\title{
Amorphous InGaZnO Thin-Film Transistors-Part I: Complete Extraction of Density of States Over the Full Subband-Gap Energy Range
}

\author{
Yongsik Kim, Minkyung Bae, Woojoon Kim, Dongsik Kong, Hyun Kwang Jeong, Hyungtak Kim, Member, IEEE,
} Sunwoong Choi, Dong Myong Kim, Member, IEEE, and Dae Hwan Kim, Member, IEEE

\begin{abstract}
A combination of the multifrequency $C-V$ and the generation-recombination current spectroscopy is proposed for a complete extraction of density of states (DOS) in amorphous InGaZnO thin-film transistors (a-IGZO TFTs) over the full subband-gap energy range $\left(E_{V} \leq E \leq E_{C}\right)$ including the interface trap density between the gate oxide and the a-IGZO active layer. In particular, our result on the separate extraction of acceptorand donor-like DOS is noticeable for a systematic design of amorphous oxide semiconductor TFTs because the former determines their dc characteristics and the latter does their threshold voltage $\left(V_{T}\right)$ instability under practical operation conditions. The proposed approach can be used to optimize the fabrication process of thin-film materials with high mobility and stability for massproduction-level amorphous oxide semiconductor TFTs.
\end{abstract}

Index Terms-Amorphous InGaZnO (a-IGZO), density of states (DOS), full subband gap, thin-film transistors (TFTs).

\section{INTRODUCTION}

$\mathbf{T}$ HE AMORPHOUS InGaZnO thin-film transistor (a-IGZO TFT) has been recognized as one of promising candidates substituting hydrogenated amorphous silicon (a-Si:H), low-temperature polycrystalline silicon, and organic TFTs as switching/driving devices in active-matrix liquid crystal displays (AMLCDs) and/or active-matrix organic light-emitting diode displays (AMOLEDs) because of its considerable merits in flexibility, visible light transparency, large-area uniformity of a low temperature sputter-deposited amorphous material, and high carrier mobility. Moreover, very

Manuscript received November 22, 2011; revised May 18, 2012 and May 31, 2012; accepted July 3, 2012. Date of publication September 4, 2012; date of current version September 18, 2012. This work was supported in part by the National Research Foundation of Korea under Grants 2012-0000147 and 20100013883 funded by the Ministry of Education, Science, and Technology, Korea, and in part by the research program of Kookmin University. The review of this paper was arranged by Editor L. Lunardi.

Y. Kim, M. Bae, W. Kim, D. Kong, H. K. Jeong, S. Choi, and D. M. Kim are with the School of Electrical Engineering, Kookmin University, Seoul 136-702, Korea.

H. Kim is with the School of Electrical Engineering, Hongik University, Seoul 121-791, Korea.

D. H. Kim is with the School of Electrical Engineering, Kookmin University, Seoul 136-702, Korea, and also temporarily with the Department of Electrical Engineering and Computer Science, University of Michigan, Ann Arbor, MI 48109 USA (e-mail: drlife@kookmin.ac.kr; daehkim@umich.edu).

Color versions of one or more of the figures in this paper are available online at http://ieeexplore.ieee.org.

Digital Object Identifier 10.1109/TED.2012.2208969 recently, various display backplanes driven by a-IGZO TFTs have been successfully demonstrated [1]-[3].

However, in spite of various demonstrations, the bias/ temperature/photo-illumination stress-induced instabilities have emerged as challenging issues for the manufacturability of a-IGZO TFTs [4], [5]. From these viewpoints, the most critical issue for a successful mass production through a systematic design of the amorphous oxide semiconductor (AOS) TFT is a comprehensive understanding of the density of states (DOS) over the full subband-gap energy range $\left(E_{V} \leq E \leq E_{C}\right)$. This is because it determines the long-term instability and the electrical characteristics. In particular, separate extraction and discrimination of each component in the subband-gap acceptor-like DOS near the conduction-band minimum (CBM; $\left.E_{C}\right)\left[g_{A}(E)\right]$ and the donor-like DOS $\left[g_{D}(E)\right]$ including one near the valence-band maximum (VBM; $\left.E_{V}\right)\left[g_{\mathrm{TD}}(E)\right]$ and the other density of shallow donor states very close to CBM $\left[g_{\mathrm{OV}}(E)\right]$ (as the process-controlled parameters) from electrical characteristics of $n$-channel a-IGZO TFTs are very important. It is because the former determines the dc performance and the latter does the threshold voltage $\left(V_{T}\right)$ instability under practical operation conditions.

To date, various methods for extracting $g_{A}(E)$ from electrical characteristics have been reported for AOS TFTs. While Jeon et al. [6] and Park et al. [7] reported the extraction technique for $g_{A}(E)$ through the optical response of $C-V$ characteristics in a-IGZO TFTs, Hsieh et al. [8] derived it by fitting the commercial technology computer-aided-design (TCAD) tool-based model with measured $I-V$ data. Chen et al. [9] also extracted $g_{A}(E)$ by applying the Meyer-Neldel rulebased model to the temperature dependence of $I-V$ characteristics. More recently, Lee et al. [10] and Bae et al. [11] proposed the $g_{A}(E)$ extraction using the frequency dependence of the measured $C-V$ characteristics and the differential ideality factor, respectively.

We also note that Nomura et al. [12] reported the $g_{\mathrm{TD}}(E)$ extracted by using X-ray photoelectron spectroscopy (XPS) measurement; however, it makes hard to apply their results to a TFT device itself because they obtained $g_{\mathrm{TD}}(E)$ from a thin-film bulk rather than TFTs. In particular, it is also difficult to expand the extracted $g_{\mathrm{TD}}(E)$ value to a practical device model, since the energy level distribution of the extracted $g_{\mathrm{TD}}(E)$ value cannot be exactly mapped to the subbandgap energy level of the a-IGZO active layer in TFT devices. 

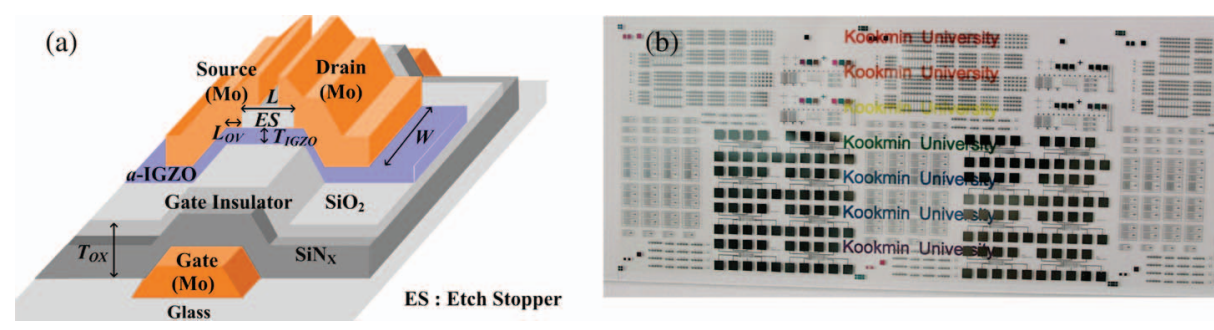

Fig. 1. (a) Schematic illustration of integrated a-IGZO TFT with the commonly used inverted staggered bottom-gate structure. (b) Photograph of a-IGZO TFTs and circuits integrated on a glass substrate.

Meanwhile, Godo et al. [13] and Fung et al. [14] extracted $g_{\mathrm{TD}}(E)$ and $g_{\mathrm{OV}}(E)$ by fitting the TCAD simulation results with measured $I-V$ data and also showed their subgap-DOSbased AOS TFT model. However, most of the parameters were employed from literatures, and the uniqueness of the extracted DOS parameters was not guaranteed. More recently, Bae et al. [15] have demonstrated the extraction technique for $g_{\mathrm{TD}}(E)$ from the measured generation-recombination $(\mathrm{G}-\mathrm{R})$ current. However, the interface states between the gate oxide and the a-IGZO active layer were excluded in calculating the G-R current, although the interface trap density is an important parameter in the long-term reliability and the performance of TFTs [16].

Consequently, there has been no report on extracting DOS over the full subband-gap energy range from the electrical characteristics of AOS TFTs, particularly under a single framework, even with disentangling the interface trap density $\left[D_{\text {it }}(E)\right]$ from the AOS thin-film bulk DOS $[g(E)]$. Motivated by these backgrounds, in this paper, both $g_{A}(E)$ and $g_{D}(E)$ of a-IGZO TFTs are completely extracted over the full subband-gap energy range with including the $D_{\text {it }}(E)$ between the gate dielectric and the a-IGZO active layer. As a novel method for separately extracting and identifying $g_{A}(E), g_{D}(E)$, and $D_{\text {it }}(E)$ from one another, a combinatory technique of multifrequency $C-V$ spectroscopy [10] and $G-R$ current spectroscopy [15] is demonstrated with the $I-V$ model based on the DOS-based amorphous oxide TFT Simulator (DeAOTS) [17].

Compared with [10], [15], and [17], $D_{\mathrm{it}}(E)$, as well as $g_{A}(E)$ and $g_{D}(E)$, is extracted and identified under a single framework. First of all, a 1-D field solver, as well as the $I_{D S}\left(V_{G S}, V_{D S}\right)$ model in [17], is modified and improved. Second, the G-R rate via interface traps is additionally incorporated into the $I_{G-R}\left(V_{G}, V_{D}\right)$ model in [15]. Finally, the approach solving $I_{G-R}\left(V_{G}, V_{D}\right)$ jointly with $I_{D S}\left(V_{G S}, V_{D S}\right)$ is proposed by using a consistent $g_{A}(E) / D_{\text {it }}(E)-\phi(x)$ relationship, where $\phi(x)$ is the electrostatic potential across the thin-film depth direction $x$. Compared with [15], it makes the accuracy and the credibility of extracted $g_{\mathrm{TD}}(E)$ and $g_{\mathrm{OV}}(E)$ significantly improved because they are extracted by using $I_{D S}\left(V_{G S}, V_{D S}\right)$ over a wide range of $V_{G S}$ and $V_{D S}$, as well as $I_{G-R}\left(V_{G}, V_{D}\right)$, while in [15], they were extracted only from $I_{G-R}\left(V_{G}, V_{D}\right)$.

\section{Device Structure and Fabrication Process}

A schematic illustration of the integrated a-IGZO TFT with a bottom-gate etch stopper structure is shown in Fig. 1 with a photograph of a-IGZO TFTs and circuits integrated on a glass substrate. Prior to the device fabrication, the substrates were chemically cleaned using aqueous mixtures of $\mathrm{H}_{2} \mathrm{SO}_{4}-\mathrm{H}_{2} \mathrm{O}_{2}$ and then rinsed with deionized water. The bottom-gate and top contact structured TFTs were fabricated using the standard semiconductor processes. The RF sputter-deposited Mo was used as the gate electrode and patterned through dry etching. A 400-nm-thick $\mathrm{SiN}_{x} / 50$-nm-thick $\mathrm{SiO}_{x}$ bilayer was used as the gate insulator and was deposited through the plasma-enhanced chemical vapor deposition (PECVD) at $370{ }^{\circ} \mathrm{C}$. For the a-IGZO (In:Ga:Zn = 2:2:1) active thin-film layer deposition, IGZO targets were prepared by conventional solid-state sintering. The 50-nm-thick IGZO active thin film was deposited by the dc sputtering at room temperature in a gas mixture of $\mathrm{Ar} / \mathrm{O}_{2}=$ 35/63 (at sccm) and patterned by the wet etch process with a diluted HF. During the sputtering, the chamber pressure was maintained at 5 mtorr and the dc power at $80 \mathrm{~W}$. Then, the 50-nm-thick etch stopper $\mathrm{SiO}_{x}$ layer was deposited by PECVD and patterned by dry etching. For the formation of source/drain (S/D) electrodes, Mo was dc sputter deposited and then patterned by a dry etching. Subsequently, the passivation layers (100-nm-thick $\mathrm{SiO}_{x}$ and 100-nm-thick $\mathrm{SiN}_{x}$ ) were deposited by PECVD at $280{ }^{\circ} \mathrm{C}$. Finally, fabricated TFTs were annealed at $250^{\circ} \mathrm{C}$ for $1 \mathrm{~h}$ in the furnace.

The fabricated a-IGZO TFT has a gate dielectric thickness $T_{\mathrm{OX}}=258 \mathrm{~nm}$ as an equivalent oxide thickness $(400 / 50 \mathrm{~nm}$ of the $\mathrm{SiN}_{X} / \mathrm{SiO}_{X}$ bilayer), the channel width $W=200 \mu \mathrm{m}$, the channel length $L=100 \mu \mathrm{m}$, the gate-to-S/D overlap length $L_{\mathrm{ov}}=10 \mu \mathrm{m}$, and the thickness of a-IGZO thin-film $T_{\mathrm{IGZO}}=$ $50 \mathrm{~nm}$. Typical device parameters were obtained to be the ON current $I_{D S}=0.86[\mu \mathrm{A}]$ at $V_{G S}=V_{D S}=10.1 \mathrm{~V}, V_{\mathrm{ON}}=$ $3.05[\mathrm{~V}]\left(V_{\mathrm{ON}}\right.$ was defined as $V_{G S}$ where a sharp increase in $I_{D S}$ occurs), the threshold voltage $V_{T}=5.3[\mathrm{~V}]$ (defined as $V_{G S}$ at $I_{D S}=1 \mathrm{nA}$ in the transfer curve), the subthreshold swing $(\mathrm{SS})=0.84[\mathrm{~V} / \mathrm{dec}]$, the field-effect mobility $\left(\mu_{\mathrm{FE}}\right)=$ $8.1\left[\mathrm{~cm}^{2} / \mathrm{Vs}\right]$ at $V_{G S}-V_{T}=15 \mathrm{~V}$, and the $I_{\mathrm{ON}} / I_{\mathrm{OFF}}$ ratio $=$ $1.7 \times 10^{6}$.

\section{Complete Extraction of DOS OVer the Full SUBBAND-GAP ENERGY RANGE}

Fig. 2 schematically illustrates the distribution of subbandgap DOS in AOS thin-film materials. Among subband-gap DOS parameters of a-IGZO TFTs, $g_{A}(E)$ has been extracted without any precondition in its mathematical form and reproducibly fitted very well with the superposition of two exponential formulas [6], [9], [10], which are consistent with cases 


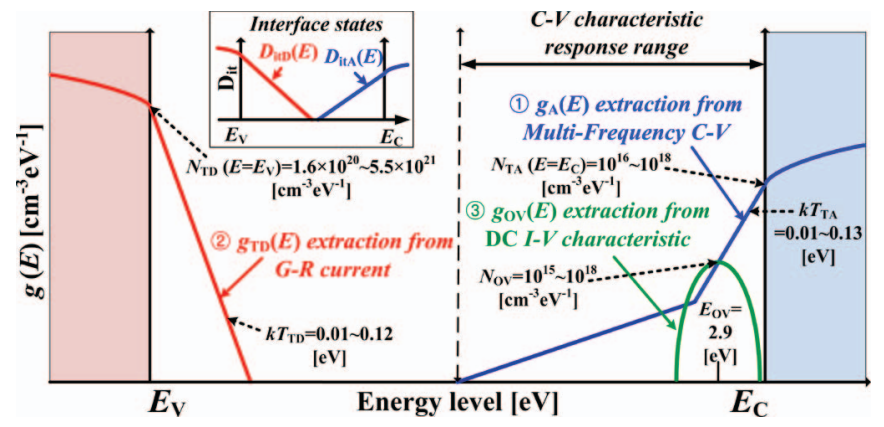

Fig. 2. Schematic illustration of the subband-gap DOS in AOS thin-film materials. It consists of the acceptor-like DOS $g_{A}(E)$ (to be extracted from the multifrequency $C-V$ spectroscopy in Section III-A), the donor-like tail state $\operatorname{DOS} g_{\mathrm{TD}}(E)$ (to be extracted from the $G-R$ current $\left(I_{G-R}\right)$ spectroscopy in Section III-B), and the shallow donor state $g_{\mathrm{OV}}(E)$ (to be extracted by fitting all of the calculated $I_{D S}-V_{G S}, I_{D S}-V_{D S}$, and $I_{G-R}-V_{G}$ characteristics with the measured ones, as described in Section III-C). (Inset) Schematic illustration of the interface trap density $D_{\text {it }}(E)$.

of a-Si:H TFTs [18], [19]. Therefore, the density of acceptorlike tail states near $E_{C}\left[g_{\mathrm{TA}}(E)\right]$ and the density of acceptorlike deep states $\left[g_{\mathrm{DA}}(E)\right]$ are mathematically modeled in this paper as

$$
\begin{aligned}
g_{A}(E)= & g_{\mathrm{DA}}(E)+g_{\mathrm{TA}}(E) \\
= & N_{\mathrm{DA}} \times \exp \left(\frac{E-E_{C}}{k T_{\mathrm{DA}}}\right) \\
& +N_{\mathrm{TA}} \times \exp \left(\frac{E-E_{C}}{k T_{\mathrm{TA}}}\right)
\end{aligned}
$$

where $g_{\mathrm{TA}}(E)$ is known to originate from the disorder of metal cation $s$-bands, namely, In-O-metal bonding angle variation, and denotes the degree of strong dispersion of electronic structures near $E_{C}$ [20]-[22]. Meanwhile, the physical origin of $g_{\mathrm{DA}}(E)$ has not been clarified yet and has been thought to be related with the weakly bonded oxygen [23]. Although the $g_{A}(E)$ values extracted in previous works [6]-[10] are slightly different from one another depending on the process and/or the structure of TFTs, the density of acceptor-like tail states (at $E=E_{C}$ ) $N_{\mathrm{TA}}=10^{16} \sim 10^{18}\left[\mathrm{~cm}^{-3} \mathrm{eV}^{-1}\right]$ and the characteristic energy of acceptor-like tail states $k T_{\mathrm{TA}}=0.01 \sim$ $0.13[\mathrm{eV}]$ are relatively lower than those of a-Si:H TFTs $\left(N_{\mathrm{TA}}=10^{21}\left[\mathrm{~cm}^{-3} \mathrm{eV}^{-1}\right]\right.$ for a-Si:H [18]).

On the other hand, $g_{\mathrm{TD}}(E)$ is closely related to the photoelectric stress-induced instability, and $g_{\mathrm{OV}}(E)$ works as donors for free carriers. They may be mathematically modeled after [13], [18], and [19] as

$$
\begin{aligned}
g_{D}(E)= & g_{\mathrm{TD}}(E)+g_{\mathrm{OV}}(E) \\
= & N_{\mathrm{TD}} \times \exp \left(\frac{E_{V}-E}{k T_{\mathrm{TD}}}\right) \\
& +N_{\mathrm{OV}} \times \exp \left[-\left(\frac{E_{\mathrm{OV}}-E}{k T_{\mathrm{OV}}}\right)^{2}\right]
\end{aligned}
$$

where $g_{\mathrm{TD}}(E)$ originates from the oxygen $p$-band disorder and the deep donor level by oxygen vacancy $\left(V_{O}\right)$ defects having the inward relaxation with neighboring metal atoms followed by the large vacancy size and reflects the degree of strongly localized states near $E_{V}$, namely, a weak dispersion of holes. In addition, $g_{\mathrm{OV}}(E)$ originates from the shallow donor states due to $V_{O}$ defects having the outward relaxation with neighboring metal atoms followed by a small vacancy size [20]-[22].

In addition, the interface trap density $D_{\mathrm{it}}(E)$ at the interface between the AOS thin-film bulk and the gate insulator can be described as

$$
\begin{aligned}
D_{\mathrm{it}}(E)= & D_{\mathrm{itA}}(E)+D_{\mathrm{itD}}(E) \\
= & N_{\mathrm{itA}} \times \exp \left(\frac{E-E_{C}}{k T_{\mathrm{itA}}}\right) \\
& +N_{\mathrm{itD}} \times \exp \left(\frac{E_{V}-E}{k T_{\mathrm{itD}}}\right)
\end{aligned}
$$

where $D_{\mathrm{itA}}$ and $D_{\mathrm{itD}}$ denote the acceptor-like and donor-like interface trap densities, respectively. They are also shown in the inset of Fig. 2.

In this section, a technique for combining the multifrequency $C-V$ spectroscopy [10], the G-R current $I_{G-R}$ spectroscopy modified from [15] with the improved DeAOTS from [17] is proposed for the extraction and the identification of $D_{\mathrm{it}}(E)$, as well as $g_{A}(E)$ and/or $g_{D}(E)$ from electrical characteristics of a-IGZO TFTs. To the best of our knowledge, our result is the first demonstration of extracting the subband-gap DOS over a full subband-gap energy range from electrical characteristics of AOS TFTs in a single frame.

\section{A. Multifrequency $C-V$ Spectroscopy for Extracting $g_{A}(E)$}

The extraction of $g_{A}(E)$ is based on the previously reported multifrequency $C-V$ spectroscopy [10]. Figs. 3(a)-(d) shows a $g_{A}(E)$-extraction procedure initiated by measuring the frequency-dependent $C-V$ curves at three different frequencies ( $f=f_{1}, f_{2}$, and $f_{3}$ ), as shown in Fig. 3(e). The frequencydependent $C-V$ curves were measured by using HP $4284 L C R$ meter at room temperature under dark conditions.

As already introduced in [10], just a key procedure is summarized in this subsection. The multifrequency $C-V$ spectroscopy is based on a postulation that the measured $C-V$ characteristic appears to be frequency dependent due to the frequency dispersion originated from the equivalent $R C$ network in Fig. 3(c). Here, $C_{\mathrm{ox}}, C_{\mathrm{LOC}}, R_{\mathrm{LOC}}$, and $C_{\mathrm{FREE}}$ are the gate dielectric capacitance, the capacitance due to the $V_{G S}$-responsive localized charges trapped in $g_{A}(E)\left[Q_{\mathrm{LOC}}\right]$, the equivalent resistance reflecting the retardation of $V_{G S}$-responsive $Q_{\mathrm{LOC}}$ (i.e., dispersions of capture-emission process of electrons), and the capacitance due to $V_{G S}$-responsive free-electron charge in the conduction band $\left(E>E_{C}\right)\left[Q_{\mathrm{FREE}}\right]$ (see the $C-V$ responsive range denoted in Fig. 2), respectively. Here, it is assumed that all of $C_{\mathrm{FREE}}, C_{\mathrm{LOC}}$, and $R_{\mathrm{LOC}}$ are independent of $f$ but depends on $V_{G S}\left(C_{\mathrm{ox}}\right.$ is independent of both $f$ and $\left.V_{G S}\right)$.

With incorporating the measured $R_{S}\left(V_{G S}\right)$ value in Fig. 3(f) into Fig. 3(b) and (c), the frequency-independent $C_{G}-V_{G S}$ characteristic $\left[C_{G, \mathrm{FI}}\left(V_{G S}\right)\right]$ was obtained, as shown in Fig. 3(g), by applying the model in Fig. 3(d) to $C_{G, F I}\left(V_{G S}\right)$. Furthermore, not only $C_{G, \mathrm{FI}}\left(V_{G S}\right)$ but also $C_{\mathrm{FREE}}\left(V_{G S}\right)$ and $C_{\mathrm{LOC}}\left(V_{G S}\right)$ can be found, as shown in the inset of Fig. 3(g). Noticeably, it is found that the crossover of dominant capacitance (from $C_{\mathrm{LOC}}$ to $C_{\mathrm{FREE}}$ ) is very clearly observed 


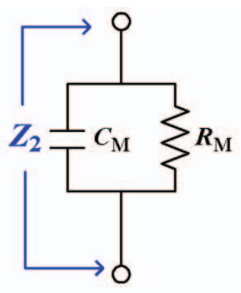

(a)
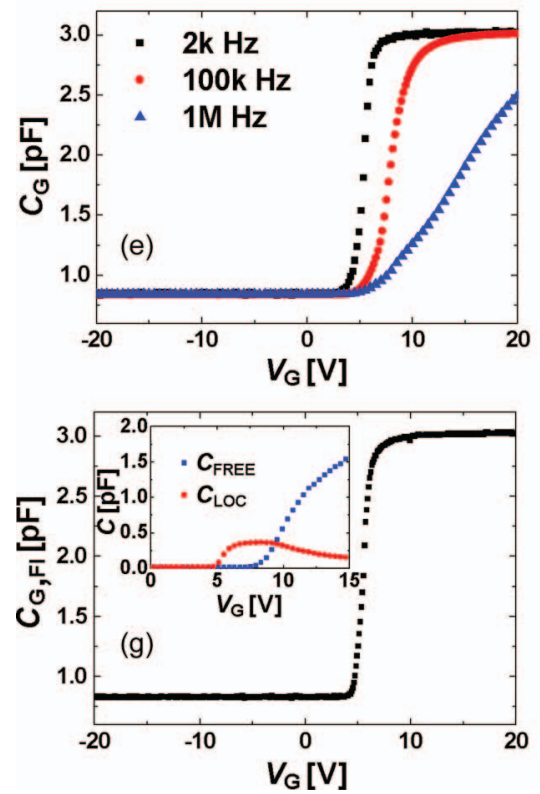

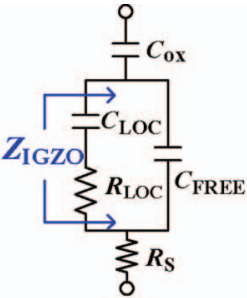

(c)

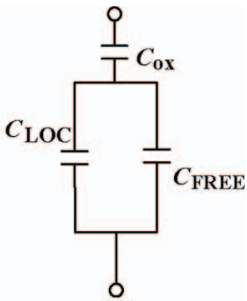

(d)
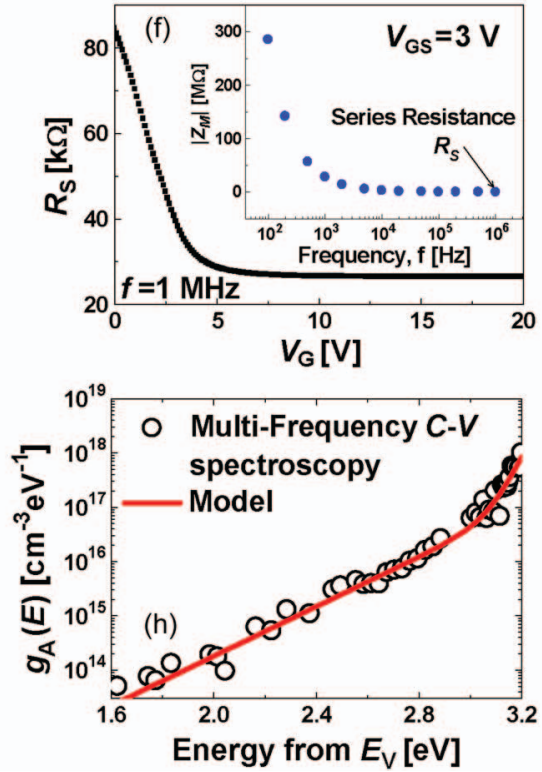

Fig. 3. Procedure of extracting $g_{A}(E)$ based on the multifrequency $C-V$ spectroscopy. (a) Two-element capacitance model for the parallel-mode measurement of an $L C R$ meter. (b) Four-element capacitance model for the de-embedding of $C_{\mathrm{OX}}$ and $R_{S}$. (c) Physics-based capacitance model with $C_{\mathrm{LOC}}, R_{L}$, and $C_{\mathrm{FREE}}$. (d) Equivalent model of the frequency-independent $C_{G}$ described by $C_{\mathrm{ox}}, C_{\mathrm{LOC}}$, and $C_{\mathrm{FREE}}$. (e) Measured frequency-dependent $C_{G}-V_{G S}$ characteristics. (f) Extracted $V_{G S}$-dependent S/D series resistance $R_{S}$ obtained from the saturated magnitude of $Z_{2}$ at high frequency in the two-element model under a fixed $V_{G S}$ value, as shown in the inset. (g) Frequency-independent $C_{G, \mathrm{FI}}-V_{G S}$ characteristic obtained by using the multifrequency $C-V$ spectroscopy. (Inset) Extracted $C_{\mathrm{LOC}}$ and $C_{\mathrm{FREE}}$. (h) (symbol) Finally extracted $g_{A}(E)$ and (line) its mathematical model that is composed of two exponential energy distributions $\left[g_{\mathrm{TA}}(E)\right.$ and $\left.g_{\mathrm{DA}}(E)\right]$.

around $V_{G S}=9.45 \mathrm{~V}$ [corresponding to the electron quasi-Fermi level $\left(E_{\mathrm{Fn}}\right)=E_{C}-0.06 \mathrm{eV}$ at the interface] that we can disentangle $Q_{\mathrm{LOC}}$ and $Q_{\mathrm{FREE}}$ by using $C_{\mathrm{LOC}}\left(V_{G S}\right)$ and $C_{\mathrm{FREE}}\left(V_{G S}\right)$ even when $E_{\mathrm{Fn}}$ is located in $g_{\mathrm{TA}}(E)$.

The $C_{\mathrm{LOC}}\left(V_{G S}\right)$ then provides information on DOS, and it can be extracted by using $Z_{2}=Z_{4}, Z_{\mathrm{CH}}=Z_{\mathrm{IGZO}}$, and $R_{\mathrm{LOC}}\left(f_{1}\right)=R_{\mathrm{LOC}}\left(f_{2}\right)=R_{\mathrm{LOC}}\left(f_{3}\right)$. In this paper, $f_{1}=$ $2 \mathrm{kHz}, f_{2}=100 \mathrm{kHz}$, and $f_{3}=1 \mathrm{MHz}$ were used as three frequencies, as shown in Fig. 3(e). Finally, $g_{A}(E)$ was extracted, as shown in the symbol of Fig. 3(h), which is obtained from $C_{\mathrm{LOC}}\left(V_{G S}\right)$ [inset of Fig. 3(g)] combined with (4) and (5) as follows:

$$
\begin{aligned}
g_{A}(E) & =g_{A}\left(V_{G S}\right)=g_{A}\left(\phi_{S}\right)=\frac{\Delta C_{\mathrm{LOC}}}{q^{2} \times W \times L \times T_{\mathrm{IGZO}}} \\
& =\frac{\left[C_{\mathrm{LOC}}\left(V_{G S 1}\right)-C_{\mathrm{LOC}}\left(V_{G S 2}\right)\right]}{q^{2} \times W \times L \times T_{\mathrm{IGZO}}} \\
\phi_{s} & =\int_{V_{\mathrm{FB}}}^{V_{G S}}\left(1-\frac{C_{G, \mathrm{FI}}\left(V_{G S}\right)}{C_{\mathrm{ox}}}\right) d V_{G S} .
\end{aligned}
$$

As aforementioned in (1), the line in Fig. 3(h) shows that the extracted $g_{A}(E)$ [symbols in Fig. 3(h)] fits very well to
TABLE I

Extracted Parameters by Using the Procedure In Fig. 7

\begin{tabular}{ccl|ccl}
\hline Parameter & Value & Unit & Parameter & Value & Unit \\
\hline$N_{\mathrm{C}}$ & $1 \times 10^{18}$ & {$\left[\mathrm{~cm}^{-3}\right]$} & $k T_{\mathrm{TD}}$ & 0.10 & {$[\mathrm{eV}]$} \\
$N_{\mathrm{V}}$ & $4.5 \times 10^{21}$ & {$\left[\mathrm{~cm}^{-3}\right]$} & $N_{\mathrm{itA}}$ & $2.38 \times 10^{12}$ & {$\left[\mathrm{~cm}^{-2} \mathrm{eV}^{-1}\right]$} \\
$\mu_{\text {Band }}$ & 9.15 & {$\left[\mathrm{~cm}^{2} / \mathrm{V} \cdot \mathrm{s}\right]$} & $k T_{\mathrm{itA}}$ & 0.19 & {$[\mathrm{eV}]$} \\
$N_{\mathrm{TA}}$ & $1 \times 10^{18}$ & {$\left[\mathrm{~cm}^{-3} \mathrm{eV}^{-1}\right]$} & $N_{\mathrm{itD}}$ & $2.25 \times 10^{16}$ & {$\left[\mathrm{~cm}^{-2} \mathrm{eV}^{-1}\right]$} \\
$k T_{\mathrm{TA}}$ & 0.03 & {$\left[\mathrm{eV}^{-1}\right]$} & $k T_{\mathrm{itD}}$ & 0.10 & {$[\mathrm{eV}]$} \\
$N_{\mathrm{DA}}$ & $1 \times 10^{17}$ & {$\left[\mathrm{~cm}^{-3} \mathrm{eV}^{-1}\right]$} & $n_{\mathrm{i}}^{2}$ & $2.92 \times 10^{4}$ & {$\left[\mathrm{~cm}^{-6}\right]$} \\
$k T_{\mathrm{DA}}$ & 0.19 & {$\left[\mathrm{eV}^{-1}\right]$} & $\sigma$ & $1 \times 10^{-14}$ & {$\left[\mathrm{~cm}^{2}\right]$} \\
$N_{\mathrm{OV}}$ & $1 \times 10^{16}$ & {$\left[\mathrm{~cm}^{-3} \mathrm{eV}^{-1}\right]$} & $v_{\text {th }}$ & $1.636 \times 10^{7}$ & {$[\mathrm{~cm} / \mathrm{s}]$} \\
$k T_{\mathrm{OV}}$ & 0.1 & {$[\mathrm{eV}]$} & $W / L$ & $200 / 100$ & {$[\mu \mathrm{m}]$} \\
$E_{\mathrm{OV}}$ & 2.9 & {$[\mathrm{eV}]$} & $T_{\mathrm{IGZO}}$ & 50 & {$[\mathrm{~nm}]$} \\
$N_{\mathrm{TD}}$ & $4.5 \times 10^{21}$ & {$\left[\mathrm{~cm}^{-3} \mathrm{eV}^{-1}\right]$} & $T_{\mathrm{OX}}$ & 258 & {$[\mathrm{~nm}]$} \\
\hline
\end{tabular}

the mathematical form of (1). Their characteristic parameters are obtained to be $N_{\mathrm{TA}}=1 \times 10^{18}\left[\mathrm{eV}^{-1} \mathrm{~cm}^{-3}\right], N_{\mathrm{DA}}=1 \times$ $10^{17}\left[\mathrm{eV}^{-1} \mathrm{~cm}^{-3}\right], k T_{\mathrm{TA}}=0.03[\mathrm{eV}]$, and $k T_{\mathrm{DA}}=0.19[\mathrm{eV}]$, as summarized in Table I. 


\section{B. $G-R$ Current Spectroscopy for Extracting $g_{\mathrm{TD}}(E)$}

It should be noted that almost all of the $g_{\mathrm{TD}}(E)$ states in $n$-channel AOS TFTs would be filled with electrons under most practical operation conditions since $E_{\mathrm{Fn}}$ is quite close to $E_{C}$ even under a thermal equilibrium. It means that $g_{\mathrm{TD}}(E)$ states are electrically neutral, and therefore, a change in $g_{\mathrm{TD}}(E)$ would not affect dc $I-V$ characteristics. As expected, the transfer curve calculated by using DeAOTS is independent of the change in $g_{\mathrm{TD}}(E)$, as shown in Fig. 4. Therefore, $g_{\mathrm{TD}}(E)$ cannot be extracted by means of fitting the calculated $I-V$ characteristics with the measured ones. It means that a new characterization technique is required for extracting $g_{\mathrm{TD}}(E)$ from TFT electrical characteristics. From this viewpoint, we have noted that $g_{D}(E)$ and $g_{A}(E)$ can work as thermal G-R centers $\left[N_{t}\left(E_{t}\right)\right]$ in the Shockley-Read-Hall (SRH) G-R model and proposed the G-R current spectroscopy with its details of the physical model in [15].

In this subsection, compared with [15], the $I_{G-R}\left(V_{G}, V_{D}\right)$ model is improved in order to include the $\mathrm{G}-\mathrm{R}$ via interface traps. The details are as follows. Under the assumption that capture cross sections for electrons and holes are the same $\left(\sigma_{n}=\sigma_{p}=\sigma\left[\mathrm{cm}^{2}\right]\right)$, the electron density $n$ in the conduction band $\left(E>E_{C}\right)$, the hole density $p$ in the valence band $(E<$ $E_{V}$ ), the electron density $n^{\prime}$ at a single trap energy level $E_{t}$, the hole density $p^{\prime}$ at $E_{t}$, and the internal drain voltage $V_{D}^{\prime}$ can be expressed as

$$
\begin{aligned}
n & =N_{C} \exp \left(-\frac{E_{C}-E_{\mathrm{Fn}}-q \phi(x)}{k T}\right) \\
& =N_{C} \exp \left(-\frac{E_{C}-\left(E_{F}-q V_{D}^{\prime}\right)-q \phi(x)}{k T}\right) \\
p & =N_{V} \exp \left(-\frac{E_{\mathrm{Fp}}-E_{V}+q \phi(x)}{k T}\right) \\
& =N_{V} \exp \left(-\frac{E_{F}-E_{V}+q \phi(x)}{k T}\right) \\
n^{\prime} & =N_{C} \exp \left(-\frac{E_{C}-E_{t}}{k T}\right) \\
p^{\prime} & =N_{V} \exp \left(-\frac{E_{t}-E_{V}}{k T}\right) \\
V_{D}^{\prime} & =V_{D}-V_{\text {Schottky }}[\mathrm{V}]
\end{aligned}
$$

with $E_{F}=$ the Fermi energy level under thermal equilibrium, $E_{\mathrm{Fp}}=$ the hole quasi-Fermi level, $N_{C}=$ the effective DOS in CBM, $N_{V}=$ the effective DOS in VBM, $V_{D}=$ the applied drain voltage, and $V_{\text {Schottky }}=$ the voltage drop resulting from the Schottky contact resistance between the AOS thin-film and the drain electrode, respectively. Then, the SRH G-R rate in the TFT active film via a single level $E_{t}$, i.e., $R_{\mathrm{SRH} 1}\left(E_{t}\right)$, is described as follows:

$$
\begin{aligned}
R_{\mathrm{SRH} 1}\left(E_{t}\right) & =\sigma v_{\mathrm{th}} N_{t}\left(E_{t}\right) \frac{\left(p n-n_{i}^{2}\right)}{p+n+p^{\prime}+n^{\prime}}\left[s^{-1} \mathrm{~cm}^{-3}\right] \\
N_{t}\left(E_{t}\right) & =\int_{E_{t}}^{E_{t}+\Delta E}\left(g_{A}(E)+g_{D}(E)\right) d E\left[\mathrm{~cm}^{-3}\right] \\
n_{i}^{2} & =N_{C} N_{V} \exp \left(-\frac{E_{g, \text { eff }}}{k T}\right)
\end{aligned}
$$

with $N_{t}\left(E_{t}\right)=$ the trap density per unit volume at $E=E_{t}$, $v_{\text {th }}=$ the thermal velocity, $n_{i}=$ the intrinsic carrier density, and $E_{g \text {,eff }}=$ the effective band-gap energy of the AOS material. Also, the total SRH G-R rate in the AOS thin-film bulk $\left(R_{\mathrm{SRH} 1 \_ \text {TOT }}\right)$ through multiple levels distributed over $E_{V}<E_{t}<E_{C}$ can be obtained by integrating $R_{\mathrm{SRH} 1}\left(E_{t}\right)$ over the band gap and can be written as

$$
R_{\mathrm{SRH} 1 \_\mathrm{TOT}}=\int_{E_{V}}^{E_{C}} R_{\mathrm{SRH} 1}\left(E_{t}\right) \delta\left(E_{t}\right) d E_{t}\left[\mathrm{~s}^{-1} \mathrm{~cm}^{-3}\right]
$$

with $\delta\left(E_{t}\right)=$ the delta function of $E_{t}$. We note that $R_{\mathrm{SRH} 1 \text { TOT }}$ is a function of $x, \phi(x)$, and $V_{G}$, and the relationship between $\phi(x)$ and $V_{G}$ is exactly given by (5) in Section III-A. Therefore, the G-R current density via DOS in the AOS thin-film bulk $\left(J_{\mathrm{SRH} 1}\right)$ can be obtained by integrating $R_{\mathrm{SRH} 1 \text { тOT }}$ along the channel depth direction over $x=0 \sim$ $T_{\mathrm{IGZO}}$ as follows:

$$
J_{\mathrm{SRH} 1}=q \times \int_{0}^{T_{\mathrm{IGZO}}} R_{\mathrm{SRH} 1 \_ \text {TOT }} d x\left[\mathrm{~A} \cdot \mathrm{cm}^{-2}\right] .
$$

In addition, since the interface trap density $D_{\text {it }}(E)$ located between the AOS thin-film and the gate oxide would also act as G-R centers, i.e., $N_{\mathrm{it}}\left(E_{t}\right)$, in the same way as $N_{t}\left(E_{t}\right)$, the SRH G-R rate at the surface of the channel should be taken into account. Therefore, the $\mathrm{G}-\mathrm{R}$ current density due to $D_{\text {it }}(E)$, i.e., $J_{\mathrm{SRH} 2}$, is included in the calculation of $I_{G-R}\left(V_{G}, V_{D}\right)$ by using

$$
\begin{aligned}
R_{\mathrm{SRH} 2}\left(E_{t}\right)= & \sigma v_{\mathrm{th}} N_{\mathrm{it}}\left(E_{t}\right) \frac{n(x=0) p(x=0)-n_{i}^{2}}{n(x=0)+p(x=0)+n^{\prime}+p^{\prime}} \\
& \times\left[\mathrm{s}^{-1} \mathrm{~cm}^{-2}\right] \\
N_{\mathrm{it}}\left(E_{t}\right)= & \int_{E_{t}}^{E_{t}+\Delta E} D_{\mathrm{it}}(E) d E\left[\mathrm{~cm}^{-2}\right] \\
J_{\mathrm{SRH} 2}= & q \times \int_{E_{V}}^{E_{C}} R_{\mathrm{SRH} 2}\left(E_{t}\right) \delta\left(E_{t}\right) d E_{t}\left[\mathrm{~A} \cdot \mathrm{cm}^{-2}\right] .
\end{aligned}
$$

Consequently, the total G-R current density $J_{G-R}$ and the total $I_{G-R}$ can be calculated as

$$
\begin{aligned}
& J_{G-R}\left(V_{G}, V_{D}\right)=J_{\mathrm{SRH} 1}+J_{\mathrm{SRH} 2}\left[\mathrm{~A} / \mathrm{cm}^{2}\right] \\
& I_{G-R}\left(V_{G}, V_{D}\right)=J_{G-R} \times W \times L[\mathrm{~A}] .
\end{aligned}
$$

As shown in the calculated $N_{\mathrm{TD}}$ and/or $k_{\mathrm{TTD}}$ dependence of $I_{G-R}$ in Fig. 4(c) and (f), the height of the $I_{G-R}\left(V_{G}, V_{D}\right)$ peak with its detailed features is very sensitive to the change in $g_{\mathrm{TD}}(E)$. Meanwhile, the calculated $g_{\mathrm{OV}}(E)$ dependences are shown in Fig. 5. It is found that the change in $N_{\mathrm{OV}}$ results in the parallel shift of the $I_{G-R}\left(V_{G}, V_{D}\right)$ and $I_{D S}\left(V_{G S}, V_{D S}\right)$ curves clearly without any change in SS [see Figs. 5(a)-(d)], while $I_{D S}\left(V_{G S}, V_{D S}\right)$ is insignificantly modulated by the change in $k T_{\mathrm{OV}}$ and $E_{\mathrm{OV}}$ [see Figs. 5(e)-(i)]. From Figs. 4 and 5, 

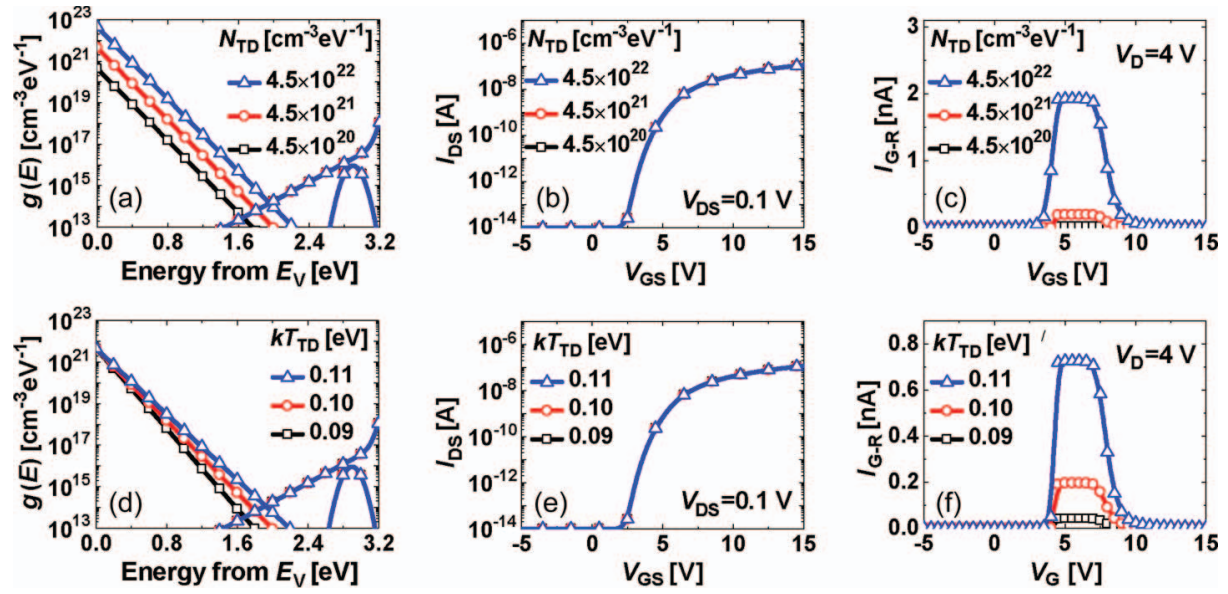

Fig. 4. (a) With a change in $N_{\mathrm{TD}}$, the (b) $I_{D S}-V_{G S}$ and (c) $I_{G-R}-V_{G}$ characteristics simulated by the modified DeAOTS. (d) With a change in $k_{\mathrm{TTD}}$, the (e) $I_{D S}-V_{G S}$ and (f) $I_{G-R}-V_{G}$ characteristics simulated by the modified DeAOTS.
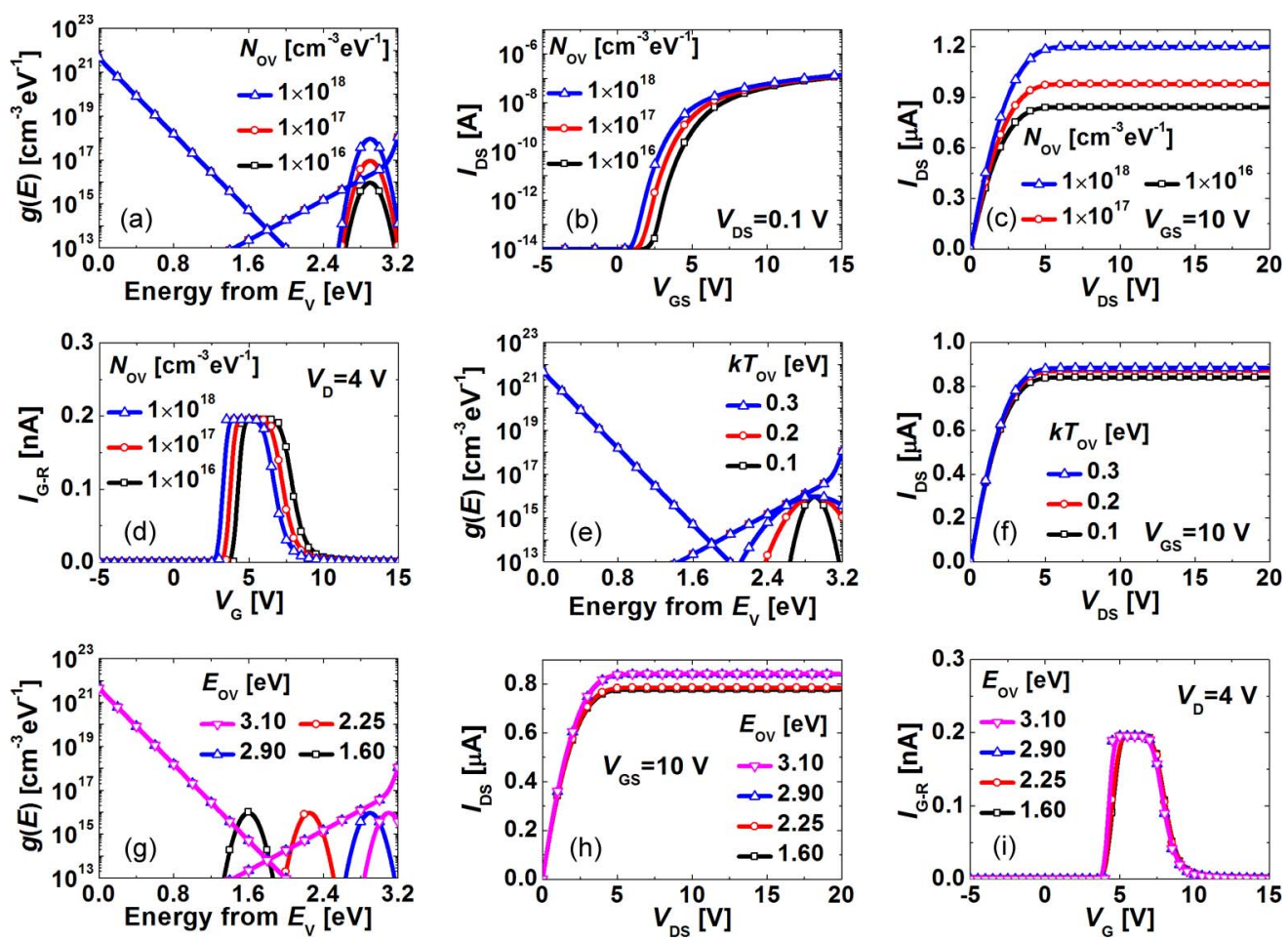

Fig. 5. (a) With a change in $N_{\mathrm{OV}}$, the (b) $I_{D S}-V_{G S}$, (c) $I_{D S}-V_{D S}$, and (d) $I_{G-R}-V_{G}$ characteristics simulated by the modified DeAOTS. (e) With a change in $k T_{\mathrm{OV}}$, (f) the $I_{D S}-V_{D S}$ characteristics simulated by the modified DeAOTS. (g) With a change in $E_{\mathrm{OV}}$, the (h) $I_{D S}-V_{D S}$ and (i) $I_{G-R}-V_{G}$ characteristics simulated by the modified DeAOTS.

it is found that $I_{D S}\left(V_{G S}, V_{D S}\right)$ depends not on $g_{\mathrm{TD}}(E)$ [as aforementioned in Fig. 4(b)] but also on $g_{A}(E)$ and $g_{\mathrm{OV}}(E)$. Therefore, $g_{\mathrm{TD}}(E)$ and $g_{\mathrm{OV}}(E)$ can be extracted by adjusting parameters (such as $N_{\mathrm{TD}}, k_{\mathrm{TTD}}, N_{\mathrm{OV}}, k T_{\mathrm{OV}}$, and $E_{\mathrm{OV}}$ ) through numerical iterations until both $I_{D S}\left(V_{G S}, V_{D S}\right)$ and $I_{G-R}\left(V_{G}, V_{D}\right)$ calculated by (24) and (20) agree well with the measured ones.

\section{Extraction of DOS Over a Full Subband-Gap Energy Range}

The $I_{D S}\left(V_{G S}, V_{D S}\right)$ characteristics were calculated by the improved DeAOTS. Compared with [17], (11) in [17] was changed for including the $D_{\text {it }}(E)$ effect as follows:

$$
\begin{aligned}
V_{G S}= & V_{\mathrm{FB}}+\left.\phi_{s}\right|_{y=y_{0}} \\
& +\left.\frac{Q_{\mathrm{loc}}(x=0)+Q_{\mathrm{free}}(x=0)+Q_{\mathrm{it}}(x=0)}{C_{\mathrm{ox}}}\right|_{y=y_{0}} \\
= & V_{\mathrm{FB}}+\left.\phi_{s}\right|_{y=y_{0}} \\
& +\left.\frac{\varepsilon_{\mathrm{IGZO}} \times E_{\mathrm{IGZO}}(x=0)+Q_{\mathrm{it}}(x=0)}{C_{\mathrm{ox}}}\right|_{y=y_{0}}
\end{aligned}
$$

with $Q_{\text {it }}(x=0)=$ the trapped charges at the interface. The 1-D field solver in [17] was also modified according to (21). 

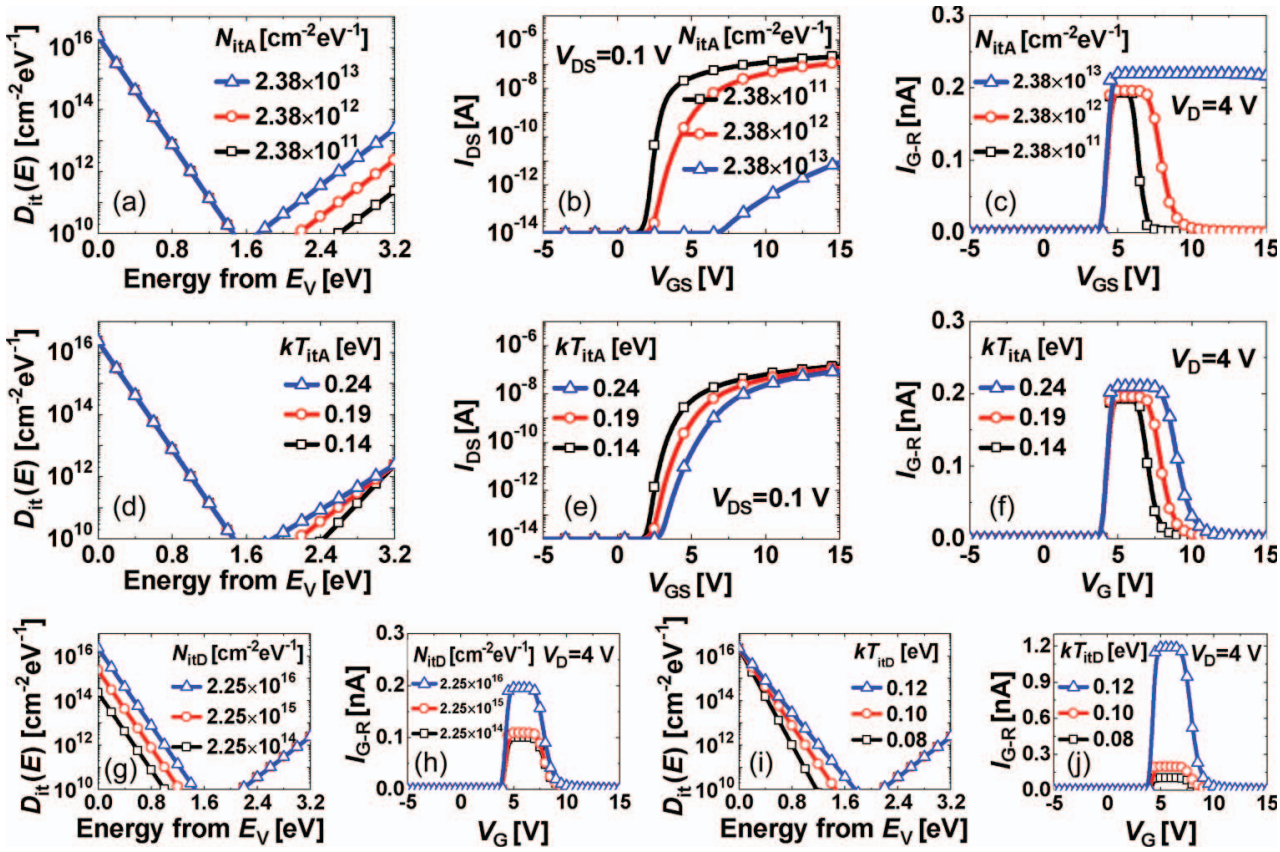

Fig. 6. (a) With a change in $N_{\text {it A }}$, the (b) $I_{D S}-V_{G S}$ and (c) $I_{G-R}-V_{G}$ characteristics simulated by the modified DeAOTS. (d) With a change in $k T_{\text {it A }}$, the (e) $I_{D S}-V_{G S}$ and (f) $I_{G-R}-V_{G}$ characteristics simulated by the modified DeAOTS. (g) With a change in $N_{\text {itD }}$, (h) the simulated $I_{G-R}-V_{G}$ characteristics. (i) With a change in $k T_{\mathrm{itD}}$, (j) the simulated $I_{G-R}-V_{G}$ characteristics.

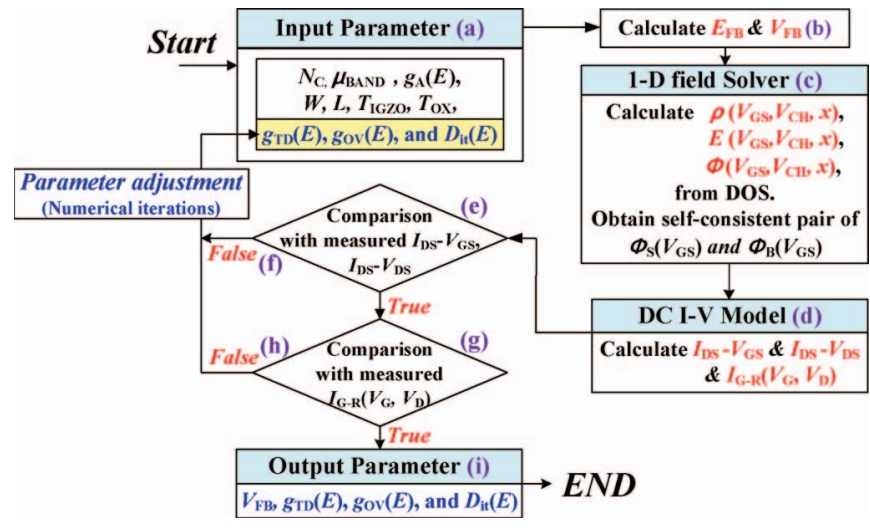

Fig. 7. Procedure of extracting DOS and $D_{\text {it }}$ parameters over the full subband-gap energy range.

Based on the improved DeAOTS, the $D_{\mathrm{itA}}$ and $D_{\mathrm{itD}}$ dependences of $I_{D S}\left(V_{G S}, V_{D S}\right)$ and $I_{G-R}\left(V_{G}, V_{D}\right)$ curves are calculated, as shown in Fig. 6. It is clearly found that $D_{\text {itA }}(E)$ mainly affects the falling tail of $I_{G-R}\left(V_{G}, V_{D}\right)$ and SS of the $I_{D S}\left(V_{G S}, V_{D S}\right)$ curve, while $D_{\text {itD }}(E)$ affects the height of $I_{G-R}\left(V_{G}, V_{D}\right)$. From the modified DeAOTS-based calculation results in Figs. 4-6, it is found that $I_{G-R}$ and $I_{D S}$ characteristics depend on both $g(E)$ and $D_{\mathrm{it}}(E)$. In detail, all of $g_{A}(E)$, $g_{\mathrm{OV}}(E), g_{\mathrm{TD}}(E), D_{\mathrm{itA}}(E)$, and $D_{\mathrm{itD}}(E)$ affect $I_{G-R}$ and $I_{D S}$ in a unique and different way from each other. Therefore, we can extract all of $g_{\mathrm{OV}}(E), g_{\mathrm{TD}}(E)$, and $D_{\mathrm{it}}(E)$ as unique solutions by adjusting them through numerical iterations until the calculated $I_{G-R}\left(V_{G}, V_{D}\right)$ and $I_{D S}\left(V_{G S}, V_{D S}\right)$ characteristics are consistent with the measured ones over a wide range of $V_{G S}$ and $V_{D S}$.

A procedure to extract $g(E)$ and $D_{\mathrm{it}}(E)$ over a full subbandgap range is shown in Fig. 7. In terms of this procedure, some parts are similar to Fig. 5 in [17]. A clear difference from [17] is that all of $g_{\mathrm{TD}}(E), g_{\mathrm{OV}}(E)$, and $D_{\mathrm{it}}(E)$ are considered by introducing $I_{G-R}$ and combining it with $I_{D S}$ in obtaining the self-consistent solutions. First of all, the fundamental input parameters including $N_{C}, N_{V}, \mu_{\text {Band }}$ (the conduction band mobility), and $g_{A}(E)$ are adopted with various geometrical parameters [e.g., $W, L, T_{\mathrm{IGZO}}$, and $T_{\mathrm{OX}}$; see Fig. 7(a)]. In this step, $N_{C}$ and $\mu_{\text {Band }}$ were chosen to be $1 \times 10^{18}\left[\mathrm{~cm}^{-3}\right]$ (by using the carrier density from the Hall measurement) and $9.15\left[\mathrm{~cm}^{2} / \mathrm{V} \cdot \mathrm{s}\right]$ (from the maximum value of measured $\mu_{\mathrm{FE}}$ ), respectively. Both are in the range consistent with previous works [14], [17], [25], [27], [28]. Also, $N_{V}$ was chosen to be $4.5 \times 10^{21}\left[\mathrm{~cm}^{-3}\right]$ by using the relationship of $N_{\mathrm{TA}} / N_{\mathrm{TD}}=$ $N_{C} / N_{V}$. Starting from all physical parameters, $V_{\mathrm{FB}}$ (flatband voltage) and $E_{\mathrm{FB}}$ (defined as the energy separation between $E_{C}$ and $E_{F}$ at $\left.V_{G S}=V_{\mathrm{FB}}\right)$ are calculated by using [see Fig. 7(b)]

$$
\begin{aligned}
V_{\mathrm{FB}} & =\phi_{\mathrm{Mo}}-\phi_{\mathrm{IGZO}}-\frac{Q_{\mathrm{ox}}}{C_{\mathrm{ox}}} \\
= & \left(\chi_{\mathrm{Mo}}-\chi_{\mathrm{IGZO}}\right)-\frac{E_{\mathrm{FB}}}{q}-\frac{Q_{\mathrm{ox}}}{C_{\mathrm{ox}}} \\
\int_{E_{V}}^{E_{C}} g_{D}(E)[1-f(E)] d E & \\
& -\int_{E_{V}}^{E_{C}} g_{A}(E) f(E) d E-n_{\text {free }}\left(E_{F}\right)=0
\end{aligned}
$$

with $\phi_{\mathrm{Mo}}=$ the work function of the gate material (Mo), $\phi_{\mathrm{IGZO}}=$ the work function of the a-IGZO layer, $\chi_{\mathrm{Mo}}=$ the electron affinity of the Mo gate, $\chi_{\mathrm{IGZO}}=$ the electron affinity of the a-IGZO layer, $\quad Q_{\mathrm{ox}}=$ the effective charge density per unit area in the gate dielectric, and $f(E)=$ the FermiDirac distribution function. Then, self-consistent solution pairs of $\phi_{B}$ (back-surface potential $\phi\left(x=T_{\mathrm{IGZO}}\right)$ ), $\phi_{S}$ (front-surface 

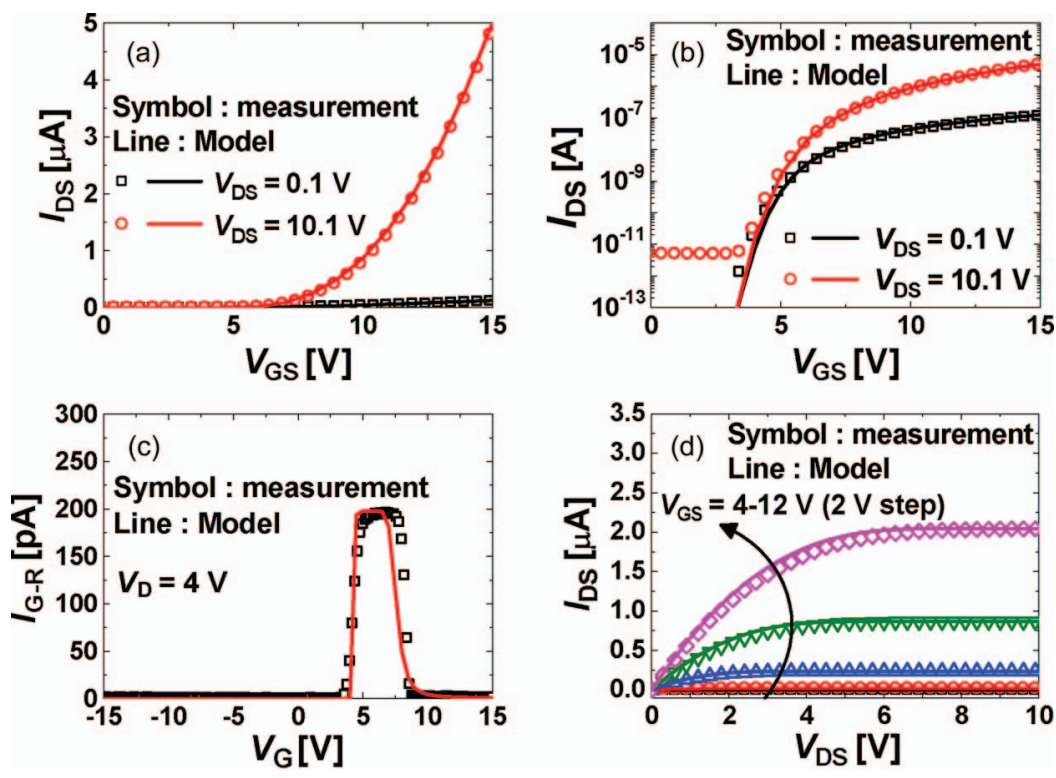

Fig. 8. Measured (a) $I_{D S}-V_{G S}$ curve in linear scale, (b) $I_{D S}-V_{G S}$ curve in log scale, (c) $I_{G-R}-V_{G}$ curve, and (d) $I_{D S}-V_{D S}$ curve of a-IGZO TFTs compared with the ones calculated by using the extracted parameter set in Table I.
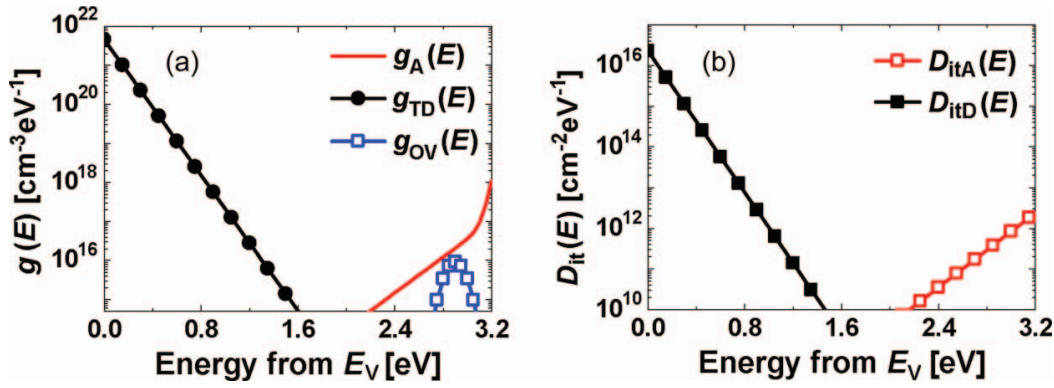

Fig. 9. $g(E)$ and $D_{\text {it }}(E)$ extracted by the procedure in Fig. 7. (a) $g(E)$ of a-IGZO thin-film bulk and (b) $D_{\text {it }}$ between the gate dielectric and a-IGZO thin-film.

potential $\phi(x=0)$ ), and $V_{G S}$ are obtained through the 1-D field solver with (21) [see Fig. 7(c)]. Then, calculations of $I_{D S}\left(V_{G S}, V_{D S}\right)$ and $I_{G-R}\left(V_{G}, V_{D}\right)$ are completed by using (20) and (24) [see Fig. 7(d)] and compared with the measured $I_{D S}\left(V_{G S}, V_{D S}\right)$ and $I_{G-R}\left(V_{G}, V_{D}\right)$ characteristics [see Fig. 7(e) and (g)]. Here, the detailed procedure of deriving (24) is given in [17], and some differences including (21) and (24) will be explained more in detail in another work, i.e.,

$$
\begin{aligned}
I_{D S}= & q \mu_{\mathrm{Band}} \frac{W}{L} \int_{V_{S}}^{V_{S}+V_{D} \phi_{B}} \int_{\phi_{S}} n_{\mathrm{FREE}}\left(\phi, V_{\mathrm{CH}}(y)\right) \\
& \times d \phi\left(-\frac{d x}{d \phi}\right) d V_{\mathrm{CH}}(y) \\
= & q \mu_{\mathrm{Band}} \frac{W}{L} \int_{V_{S}}^{V_{S}+V_{D}} \int_{\phi_{B}}^{\phi_{S}} \frac{n_{\mathrm{FREE}}\left(\phi, V_{\mathrm{CH}}(y)\right)}{E_{\mathrm{IGZO}}\left(\phi, V_{\mathrm{CH}}(y)\right)} \\
& \times d \phi d V_{\mathrm{CH}}(y) .
\end{aligned}
$$

Then, $g_{\mathrm{TD}}(E), g_{\mathrm{OV}}(E)$, and $D_{\mathrm{it}}(E)$ parameters are adjusted by numerical iterations, until the calculated $I_{D S}\left(V_{G S}, V_{D S}\right)$ and $I_{G-R}\left(V_{G}, V_{D}\right)$ characteristics agree well with the measured ones [see Figs. 7(e)-(h)]. When this matching is accomplished with the proposed parameter-extraction methodology, all parameters $\left(N_{\mathrm{TD}}, k_{\mathrm{TTD}}, N_{\mathrm{OV}}, k T_{\mathrm{OV}}, E_{\mathrm{OV}}, N_{\mathrm{itA}}, k T_{\mathrm{itA}}\right.$, $N_{\text {itD }}$, and $k T_{\text {itD }}$ ) can be extracted as a unique solution [see Fig. 7(i)] with numerical iteration under the conditions of specific error factor, significant digit, and proper range. Here, it should be reminded that $g_{A}(E)$ is not a fitting parameter because it was already known by the multifrequency $C-V$ spectroscopy in Section III-A. Table I summarizes geometrical and DOS model parameters, which are extracted by the proposed procedure in Fig. 7.

A quantitative self-consistency with experimental data is automatically guaranteed in the proposed DOS-extracting procedure. As expected, the calculated $I_{D S}\left(V_{G S}, V_{D S}\right)$ and $I_{G-R}\left(V_{G}, V_{D}\right)$ values agree very well with the measured ones in Figs. 8(a)-(d). Finally, extracted $g(E)$ and $D_{\text {it }}(E)$ values over a full subband-gap energy range are shown in Fig. 9.

Our results verify that all of the extracted parameter set, the implemented $I_{D S}\left(V_{G S}, V_{D S}\right)$ and $I_{G-R}\left(V_{G}, V_{D}\right)$ models, and the parameter-extracting methodology can successfully reproduce the measured electrical characteristics even for the subthreshold region, as well as for $V_{G S}>V_{T}$. Furthermore, the proposed method can be a main step triggering the process optimization, the device characterization, and the modeling for a systematic design and implementation of AOS TFT circuits and applications because all of $N_{C}, \mu_{\mathrm{Band}}, g_{A}(E), g_{\mathrm{TD}}(E)$, $g_{\mathrm{OV}}(E)$, and $D_{\mathrm{it}}(E)$ are process-controlled parameters. 


\section{CONCLUSION}

We have presented a method for completely extracting DOS and $D_{\text {it }}(E)$ over the full subband-gap energy range from the measured electrical characteristics of a-IGZO TFTs under a single framework. The combination of the multifrequency $C-V$ spectroscopy and the G-R current spectroscopy has been proposed and demonstrated with the modified DeAOTS model.

As process-controlled parameters, our result on separately extractable acceptor-like and donor-like DOS is noticeable for a systematic design of AOS TFTs because the former determines their dc characteristics and the latter does their instabilities in $V_{T}$ under practical operation conditions. Our approach can be used to optimize the fabrication process of thin-film materials with high mobility and stability for mass-production-level AOS TFTs, which will be innovatively used in the near future.

Based on the same theoretical model and DOS-extraction methodology, the negative-bias illumination-stress-induced $V_{T}$ instability of a-IGZO TFTs is quantitatively analyzed in Part II of this paper [29].

\section{REFERENCES}

[1] Y. Ohta, Y. Chikama, T. Hara, Y. Mizuno, T. Aita, M. Takei, M. Suzuki, O. Nakagawa, Y. Harumoto, H. Nishiki, and N. Kimura, "Amorphous In-Ga-Zn-O TFT-LCDs with high reliability," in Proc. Int. Display Workshop, Dec. 2009, pp. 1685-1688.

[2] J. Sakata, H. Ohara, M. Sasaki, T. Osada, H. Miyake, H. Shishido, J. Koyama, Y. Oikawa, H. Maruyama, M. Sakakura, T. Serikawa, and S. Yamazaki, "Development of 4.0-in. AMOLED display with driver circuit using amorphous In-Ga-Zn-Oxide TFTs," in Proc. Int. Display Workshop, Dec. 2009, pp. 689-692.

[3] J.-H. Lee, D.-H. Kim, D.-J. Yang, S.-Y. Hong, K.-S. Yoon, P.-S. Hong, C.-O. Jeong, H.-S. Park, S. Y. Kim, S. K. Lim, and S. S. Kim, "World's largest (15-inch) XGA AMLCD panel using IGZO oxide TFT," SID Dig. Tech. Papers, vol. 39, no. 1, pp. 625-628, May 2008.

[4] M. E. Lopes, H. L. Gomes, M. C. R. Medeiros, P. Barquinha, L. Pereira, E. Fortunato, R. Martins, and I. Ferreira, "Gate-bias stress in amorphous oxide semiconductors thin-film transistors," Appl. Phys. Lett., vol. 95, no. 6, pp. 063502-1-063502-3, Oct. 2009.

[5] J.-Y. Huh, S.-B. Seo, H.-S. Park, J.-H. Jeon, H.-H. Choe, K.-W. Lee, J.-H. Seo, M.-K. Ryu, S.-H. K. Park, and C.-S. Hwang, "Light and bias stability of a-IGZO TFT fabricated by r.f. magnetron sputtering," Current Appl. Phys., vol. 11, no. 5, pp. S49-S53, Sep. 2011.

[6] K. Jeon, C. Kim, I. Song, J. Park, S. Kim, S. Kim, Y. Park, J.-H. Park, S. Lee, D. M. Kim, and D. H. Kim, "Modeling of amorphous InGaZnO thin-film transistors based on the density of states extracted from the optical response of capacitance-voltage characteristics," Appl. Phys. Lett., vol. 93, no. 18, pp. 182 102-1-182 102-3, Nov. 2008.

[7] J.-H. Park, K. Jeon, S. Lee, S. Kim, S. Kim, I. Song, C. J. Kim, J. Park, Y. Park, D. M. Kim, and D. H. Kim, "Extraction of density of states in amorphous GaInZnO thin-film transistors by combining an optical charge pumping and capacitance-voltage characteristics," IEEE Electron Device Lett., vol. 29, no. 12, pp. 1292-1295, Dec. 2008.

[8] H.-H. Hsieh, T. Kamiya, K. Nomura, H. Hosono, and C.-C. Wu, "Modeling of amorphous InGaZnO4 thin film transistors and their subgap density of states," Appl. Phys. Lett., vol. 92, no. 13, pp. 133 503-1-133 503-3, Apr. 2008.

[9] C. Chen, K. Abe, H. Kumomi, and J. Kanicki, "Density of states of a-InGaZnO from temperature-dependent field-effect studies," IEEE Trans. Electron Devices, vol. 56, no. 6, pp. 1177-1183, Jun. 2009.

[10] S. Lee, S. Park, S. Kim, Y. W. Jeon, K. Jeon, J.-H. Park, J. Park, I. Song, C. J. Kim, Y. Park, D. M. Kim, and D. H. Kim, "Extraction of subgap density of states in amorphous InGaZnO thin film transistors by using multi-frequency capacitance-voltage characteristics," IEEE Electron Device Lett., vol. 31, no. 3, pp. 231-233, Mar. 2010.

[11] M. Bae, D. Yun, Y. Kim, D. Kong, H. K. Jeong, W. Kim, J. Kim, I. Hur, D. H. Kim, and D. M. Kim, "Differential ideality factor technique for extraction of subgap density of states in amorphous InGaZnO thinfilm transistors," IEEE Electron Device Lett., vol. 33, no. 3, pp. 399-401, Mar. 2012.
[12] K. Nomura, T. Kamiya, H. Yanagi, E. Ikenaga, K. Yang, K. Kobayashi, M. Hirano, and H. Hosono, "Subgap states in transparent amorphous oxide semiconductor, In-Ga-Zn-O, observed by bulk sensitive X-ray photoelectron spectroscopy," Appl. Phys. Lett., vol. 92, no. 20, pp. 202 117-1-202 117-3, May 2008.

[13] H. Godo, D. Kawae, S. Yoshitomi, T. Sasaki, S. Ito, H. Ohara, A. Miyanaga, and S. Yamazaki, "Numerical analysis on temperature dependence of characteristics of amorphous In-Ga-Zn-oxide TFT," SID Dig. Tech. Papers, vol. 40, no. 1, pp. 1110-1112, Jun. 2009.

[14] T.-C. Fung, C.-S. Chuang, C. Chen, K. Abe, R. Cottle, M. Townsend, H. Kumomi, and J. Kanicki, "Two-dimensional numerical simulation of radio frequency sputter amorphous $\mathrm{In}-\mathrm{Ga}-\mathrm{Zn}-\mathrm{O}$ thin-film transistors," J. Appl. Phys., vol. 106, no. 8, pp. 084511-1-084511-10, Oct. 2009.

[15] M. Bae, Y. Kim, S. Kim, D. M. Kim, and D. H. Kim, "Extraction of subgap donor states in a-IGZO TFTs by generation-recombination current spectroscopy," IEEE Electron Device Lett., vol. 32, no. 9, pp. 1248-1250, Sep. 2011.

[16] K. Nomura, T. Kamiya, and H. Hosono, "Interface and bulk effects for bias-light-illumination instability in amorphous-In-Ga-Zn-O thin-film transistors," J. Soc. Inf. Display, vol. 18, no. 10, pp. 789-795, Oct. 2010.

[17] Y. W. Jeon, S. Kim, S. Lee, D. M. Kim, D. H. Kim, J. Park, C. J. Kim, I. Song, Y. Park, U.-I. Chung, J.-H. Lee, B. D. Ahn, S.Y. Park, J.-H. Park, and J. H. Kim, "Subgap density-of-states-based-amorphous oxide thin film transistor simulator (DeAOTS)," IEEE Trans. Electron Devices, vol. 57, no. 11, pp. 2988-3000, Nov. 2010.

[18] J. Kanicki and S. Martin, Thin-Film Transistors, C. R. Kagan and P. Andry, Eds. New York: Marcel Dekker, 2003, pp. 71-137.

[19] R. A. Street, Hydrogenated Amorphous Silicon. Cambridge, U.K.: Cambridge Univ. Press, 1991.

[20] J. Robertson, "Physics of amorphous conducting oxides," J. Non-Cryst. Solids, vol. 354, no. 19-25, pp. 2791-2795, May 2008.

[21] T. Kamiya, K. Nomura, M. Hirano, and H. Hosono, "Electronic structure of oxygen deficient amorphous oxide semiconductor a-InGaZnO4-x: Optical analyses and first-principle calculations," Phys. Stat. Sol. (C), vol. 5 , no. 9, pp. 3098-3100, Jun. 2008.

[22] T. Kamiya, K. Nomura, and M. Hirano, "Electronic structure of the amorphous oxide semiconductor a-InGaZnO4-x:Tauc-Lorentz optical model and origins of subgap states," Phys. Stat. Sol. (A), vol. 206, no. 5, pp. 860867, Apr. 2009

[23] K. Ide, Y. Kikuchi, K. Nomura, M. Kimura, T. Kamiya, and H. Hosono, "Effects of excess oxygen on operation characteristics of amorphous In-Ga-Zn-O thin-film transistors," Appl. Phys. Lett., vol. 99, no. 9, pp. 093507-1-093507-3, Sep. 2011.

[24] T. Kamiya, K. Nomura, and H. Hosono, "Present status of amorphous In-Ga-Zn-O thin-film transistors," Sci. Technol. Adv. Mater., vol. 11, no. 4, pp. 044305-1-044305-23, Aug. 2010.

[25] T. Kamiya, K. Nomura, and H. Hosono, "Origins of high mobility and low operation voltage of amorphous oxide TFTs: Electronic structure, electron transport, defects and doping," J. Display Technol., vol. 5, no. 7, pp. $273-$ 288, Jul. 2009.

[26] J. Robertson, "Disorder and instability process in amorphous conducting oxides," Phys. Stat. Sol. (B), vol. 245, no. 6, pp. 1026-1032, Jun. 2008.

[27] P. Barquinha, A. M. Vilà, G. Gonçalves, L. Pereira, R. Martins, J. R. Morante, and E. Fortunato, "Gallium indium zinc-oxide-based thin film transistors: Influence of the source/drain material," IEEE Trans. Electron Devices, vol. 55, no. 4, pp. 954-960, Apr. 2008.

[28] A. Takagi, K. Nomura, H. Ohta, H. Yanagi, T. Kamiya, M. Hirano, and H. Hosono, "Carrier transport and electronic structure in amorphous oxide semiconductor, a-InGaZnO4," Thin Solid Films, vol. 486, no. 1/2, pp. 38 41, Aug. 2005.

[29] Y. Kim, S. Kim, W. Kim, M. Bae, H. K. Jung, D. Kong, S. Choi, D. M. Kim, and D. H. Kim, "Amorphous InGaZnO thin-film transistors-Part II: Modeling and simulation of negative bias illumination stress-induced instability," IEEE Trans. Electron Devices, vol. 59, no. 10, pp. 2699-2706, Oct. 2012.

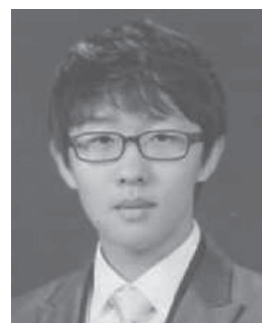

Yongsik Kim received the B.S. and M. S. degrees in electrical engineering from the Kookmin University, Korea, in 2010 and 2012, respectively. He is currently with Samsung Electronics Company, Ltd., Korea. His research interests are nanoCMOS devices and TFTs. 


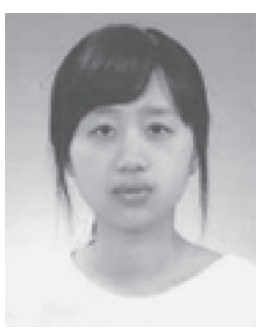

Minkyung Bae received the B.S. and M. S. degrees in electrical engineering from the Kookmin University, Korea, in 2010 and 2012, respectively. She is currently with Samsung Electronics Company, Ltd., Korea. Her research interests are nanoCMOS devices and TFTs.

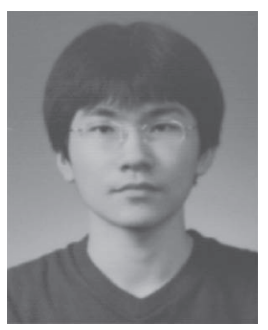

Woojoon Kim received the B.S. degree in electrical engineering from the Kookmin University, Korea, in 2011, where he is currently working toward the M.S. degree at the Department of Electrical Engineering. His research interests are oxide TFTs and nanoCMOS devices.

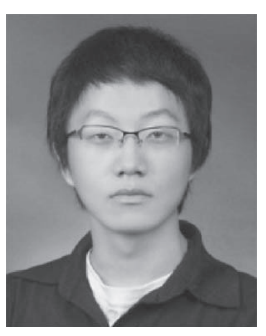

Dongsik Kong received the B.S. and M. S. degrees in electrical engineering from the Kookmin University, Korea, in 2010 and 2012, respectively. He is currently with Samsung Electronics Company, Ltd., Korea. His research interests are nanoCMOS devices and TFTs.

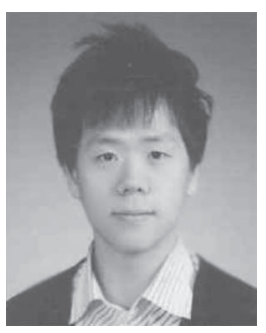

Hyun Kwang Jeong received the B.S. and M. S degrees in electrical engineering from the Kookmin University, Korea, in 2010 and 2012, respectively. $\mathrm{He}$ is currently with MagnaChip Company, Ltd., Korea. His research interests are CMOS devices and integrated circuits.
Hyungtak Kim (M'03) received the Ph.D. degree from Cornell University in 2003, in electrical engineering. He is currently an assistant professor in the school of electrical engineering at Hongik University, Korea. His research interests include reliability of wide bandgap semiconductor devices and novel TFTs.

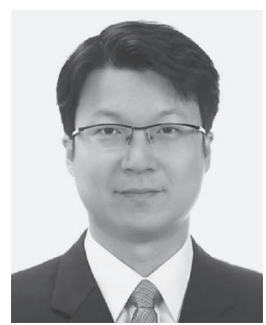

Sunwoong Choi received the PhD degree in EECS from Seoul National University in 2005. He is currently an Assistant Professor in the School of Electrical Engineering, Kookmin University, Korea. His research interests include computer networks, computer-aided engineering, and performance evaluation.

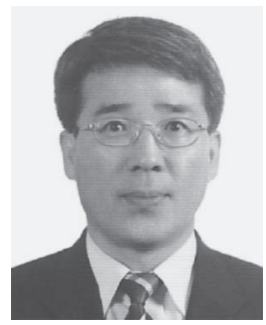

Dong Myong Kim (S'86-M'88) is currently the Professor in the School of Electrical Engineering, Kookmin University, Korea. His current research interests include fabrication, characterization, and modeling of nanostructure Silicon devices, III-V compound semiconductor devices, memories, and CMOS RF circuits.

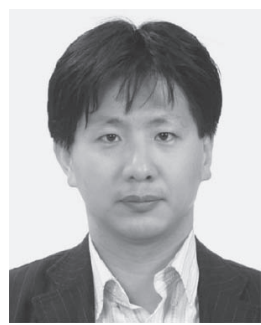

Dae Hwan Kim (M'08) is currently an Associate Professor in the School of Electrical Engineering, Kookmin University, Seoul, Korea. His research interests include nanoCMOS, TFTs, biosensors, exploratory logic and memory devices. $\mathrm{He}$ has (co)authored over 190 research publications and patents. 